\title{
Prevalence and clinical characteristics of prefrailty in elderly adults: differences according to degree of urbanization
}

\author{
Laura Lorenzo-López, Rocío López-López, Ana Maseda, Clara Diego-Díez, Sarai \\ Gómez-Caamaño, José C. Millán-Calenti
}

To the Editor: The early identification of individuals at risk of frailty is growing in importance because it may provide insights into the mechanisms involved in physical age-related decline and may help prevent the onset of disabilities and mortality.[1] Individuals with the intermediate prefrailty status have a greater risk of becoming frail.[2] Because frailty has been associated with risk of multiple adverse outcomes,[3-5] and prefrailty status seems to be partially reversible and an optimal target for interventions, [6-8] it is crucial to identify individuals who are at risk of frailty (prefrailty) to develop early and effective preventive interventions to reduce or manage the risks associated with this condition.

Populations in different geographic areas could experience different levels of frailty because of cultural or political distinctions.[9] In this context, determining prefrailty prevalence rates as a function of degree of urbanization is of particular interest because there may be considerable differences in socioeconomic status, demographic characteristics, and health care.

The prevalence and clinical characteristics of prefrailty were explored in community-dwelling elderly adults living in densely versus nondensely populated areas.

\section{Methods}

This was a cross-sectional study of a representative sample $(n=749)$ of adults aged 65 and older. Data were used from baseline assessments from the Effectiveness of the Comprehensive Gerontological Assessment and longitudinal follow-up in the healthy ageing promotion (VERISAÚDE) project. Two categories were created to define degree of urbanization: densely populated areas (DPAs, urban) and nondensely populated areas (non-DPAs, rural). Prefrailty status was diagnosed based on the Fried criteria (unintentional weight loss, exhaustion, low physical activity, low walking speed, low grip strength).[2] Sociodemographic characteristics, comorbidity, predicted 1-year mortality, drug consumption, and polypharmacy were registered.

\section{Results}

Most (71.8\%) participants were identified as prefrail, mainly as a result of low grip strength. The prevalence of prefrailty did not differ significantly between geographic areas (DPA, 71.2\%; non-DPA, $72.5 \% ; P=.92)$. In Spain, the urbanization of rural areas is increasing, and differences between areas are probably not as sharp as in other countries. A greater prevalence of prefrailty was observed in women (DPA, $P<.001$; non-DPA, $P<.001$ ) and in individuals aged 65 to 79 than in those who were older (DPA, $P<.001$; non-DPA, $P<.001$ ), but prefrailty was not associated with lower levels of education or comorbidities.

Physical capacity impairments (low grip strength, slow walking time) were more prevalent than physiological markers (exhaustion, unintentional weight loss, and low physical activity) in the prefrail population (Figure 1). Low grip strength and slow walking time were also the two most frequent physical impairments in frailty, and the proportion of all criteria except low grip strength was much higher in frail individuals, which suggests that low grip strength is not a good criterion to use to discriminate prefrail from frail individuals. It is also possible that the original Fried cutoff points[2] are not valid for a Spanish population and should be adapted to avoid inappropriate categorization. 
Proportion of single criteria in prefrail and frail groups

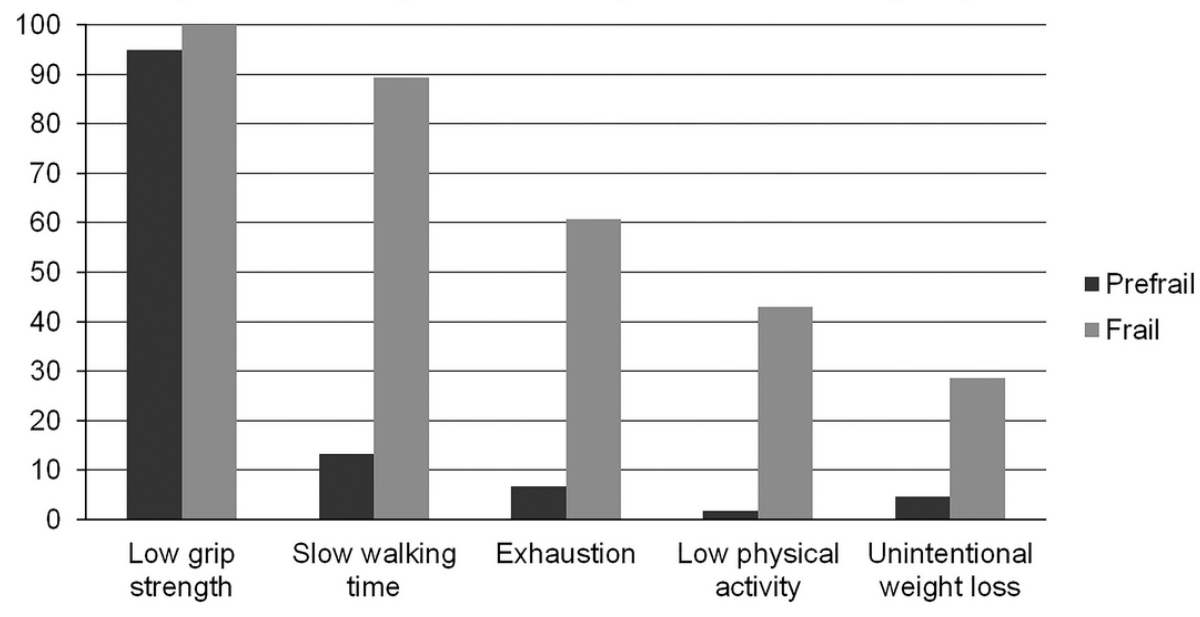

Figure 1. Proportion of single frailty criteria in prefrail and frail groups.

Mean number of drugs and polypharmacy significantly increased as a function of frailty in both areas, with prefrail individuals having intermediate consumption values (number of drugs: DPA, $P<.001$, nonDPA, $P<.001$; polypharmacy: DPA, $P=.002$, non-DPA, $P=.01$ ). The fact that many frail older adults have multiple chronic comorbidities and functional impairments and, consequently, are prescribed long lists of medications can explain this.

Although frail participants had greater comorbidity, the difference was not statistically significant (DPA, $P=.45$; non-DPA, $P=.54$ ). Mortality significantly increased as a function of frailty (again with intermediate mortality rates in prefrailty) (DPA, $P=.005$; non-DPA, $P<.001$ ). A tendency was observed in frail individuals toward higher mortality in non-DPA (39.3\%) than DPA (33.2\%).

\section{Conclusion}

A high percentage of individuals aged 65 and older are prefrail regardless of geographic setting, indicating that an important subset of community-dwelling older adults is at high risk of progressing to frailty. This is relevant because individuals who are frailty have a greater risk of poor health outcomes including falls, disability, hospitalization, and mortality.[3-5] In this context, identifiable and modifiable physical manifestations (e.g., low grip strength) may provide clinically relevant targets for developing preventive intervention strategies. Although, most studies have evaluated the effectiveness of interventions in older people who are already frail, recent evidence indicates that interventions are more effective in prefrail individuals than in those who are already frail.[10] These findings may be of longterm potential benefit to clinical elderly care.

\section{Acknowledgments}

The authors sincerely thank the staff and older adults from the 43 senior centers who contributed to this study.

This research was supported by the Xunta de Galicia project EM 2012/100: "VERISAÚDE project: Effectiveness of the Comprehensive Gerontological Assessment and longitudinal follow-up in the healthy ageing promotion."

\section{Conflict of Interest: None.}

Author Contributions: Millán-Calenti, Lorenzo-López, Maseda: concept and design. Lorenzo-López, López-López, Diego-Díez, Gómez-Caamaño: data acquisition, analysis, and interpretation. LorenzoLópez, López-López: drafting the letter. Millán-Calenti, Maseda: critical revision for important intellectual content. All authors approved the final version to be published. 
Sponsor's Role: None.

\section{References}

1. Woods NF, LaCroix AZ, Gray SL et al. Frailty: Emergence and consequences in women aged 65 and older in the Women's Health Initiative observational study. J Am Geriatr Soc2005;53:1321-1330.

2. Fried LP, Tangen CM, Walston J et al. Frailty in older adults: Evidence for a phenotype. J Gerontol A Biol Sci Med Sci 2001;56A:M146-M156.

3. Bandeen-Roche K, Xue QL, Ferrucci L et al. Phenotype of frailty: Characterization in the Women's Health and Aging Studies. J Gerontol A Biol Sci Med Sci 2006;61A:262-266.

4. Cawthon PM, Marshall LM, Michael Y et al. Frailty in older men: Prevalence, progression, and relationship with mortality. J Am Geriatr Soc 2007;55:1216-1223.

5. Song X, Mitnitski A, Rockwood K. Prevalence and 10-year outcomes of frailty in older adults in relation to deficit accumulation. J Am Geriatr Soc 2010;58:681-687.

6. Espinoza SE, Jung I, Hazuda H. Frailty transitions in the San Antonio Longitudinal Study of Aging. J Am Geriatr Soc 2012;60:652-660.

7. Fernández-Garrido J, Ruiz-Ros V, Buigues $\mathrm{C}$ et al. Clinical features of prefrail older individuals and emerging peripheral biomarkers: A systematic review. Arch Gerontol Geriatr2014;59:7-17.

8. Gill TM, Gahbauer EA, Allore HG et al. Transitions between frailty states among community-living older persons. Arch Intern Med 2006;166:418-423.

9. Santos-Eggimann B, Cuénoud P, Spagnoli J et al. Prevalence of frailty in middle-aged and older communitydwelling Europeans living in 10 countries. J Gerontol A Biol Sci Med Sci2009;64A:675-681.

10. Fairhall N, Kurrle SE, Sherrington $C$ et al. Effectiveness of a multifactorial intervention on preventing development of frailty in pre-frail older people: Study protocol for a randomised controlled trial. BMJ Open 2015;5:e07091. 